\title{
On the Finite Constellation Sum Rates for ZF and CI Precoding
}

\author{
Abdelhamid Salem and Christos Masouros \\ Department of Electronic and Electrical Engineering, \\ University College London, London, UK \\ emails: \{a.salem, c.masouros\}@ucl.ac.uk
}

\begin{abstract}
This paper analyzes the performance of multi-user multiple-input multiple-output (MU-MIMO) systems, with a finite phase-shift keying (PSK) input alphabet. The achievable sum rate is investigated for two precoding techniques, namely: 1) zero forcing ( $Z F$ ) precoding, 2) constructive interference (CI) precoding. In light of this, new analytical expressions for the average sum rate are derived in the two scenarios, and Monte Carlo simulations are provided throughout to confirm the analysis. Furthermore, based on the derived expressions, a power allocation scheme that can ensure fairness among the users is also investigated. The results in this paper demonstrate that, the CI strictly outperforms the ZF scheme, and the performance gap between the considered schemes depends essentially on the system parameters.
\end{abstract}

Index Terms-Finite constellation signaling, zero forcing, constructive interference, multiple-input multiple-output.

\section{INTRODUCTION}

D UE to their high spectral efficiency and reliability, multi-user multiple-input multiple-output (MU-MIMO) communication systems have attracted considerable attention nowadays [1]-[3]. However, the potential advantages of MUMIMO systems are often undermined by the interference in real communication systems. Consequently, considerable amount of researches have focused on mitigating the interference in MIMO systems under finite alphabet input signals. In [3] linear precoder design for interference channels of MUMIMO systems under finite alphabet inputs was investigated. In [4] the authors studied the design of linear precoders in multi-cell MIMO systems for finite alphabet signals. In [5] the capacity of a MIMO fading channel with phase-shift keying (PSK) input alphabet was analyzed. In [6], [7] the design of optimum precoders which maximize the mutual information of MIMO channels with finite-alphabet inputs were investigated. In [8], a linear precoding for MIMO channels with finite discrete inputs was studied, in which the capacity region for the MIMO channels has been derived. Although the proposed techniques in these works produced optimal performances, they have no closed form solutions and their resulting high computational complexity make them inapplicable in practical scenarios. Recently, constructive interference $(\mathrm{CI})$ precoding technique has been proposed to enhance the performance of MU-MIMO systems [9]-[20]. In contrast to the conventional techniques where the knowledge of the interference is used to cancel it, the main idea of the $\mathrm{CI}$ is to use the interference to improve the system performance. Based on the knowledge of the users' data symbols and the channel state information (CSI), the interference can be classified as constructive and destructive. The constructive interference moves the received symbols away from the decision thresholds of the constellation towards the direction of the desired symbol. Consequently, the transmit precoder can be designed to make the resulting interference is constructive to the desired symbol. This line of work has been introduced in [9], where the CI precoding scheme for the downlink of PSK-based MIMO systems has been proposed. In this work it was shown that the system performance can be enhanced by exploiting the interference signals. As a result, the effective signal to interference-plusnoise ratio (SINR) can be enhanced without the need to increase the transmitted signal power at the base station (BS). In [21] the concept of CI was used to design an optimization based precoder in the form of pre-scaling for the first time. The authors in [10] proposed transmit beamforming schemes for the MU-MIMO down-link that minimize the transmit power for PSK signals. Very recently, in [22] closed-form precoding expression for CI exploitation in the MU-MIMO down-link was derived. The closed-form precoder in this work has paved the way for the development of communication theoretic analysis of the benefits of CI, which is the focus of this work.

In this paper we analyze the sum rate of MU-MIMO systems with PSK input alphabet. In order to investigate and compare the performance of interference suppression and interference exploitation precoders, two transmission techniques are considered in this work, namely, 1) zero forcing (ZF) precoding technique, 2) CI precoding technique. In this respect, new explicit analytical expressions for the average achievable sum rate upper-bound are derived for the two transmission schemes, and Monte-Carlo simulations are provided throughout our investigation to confirm the analysis. Furthermore, based on the derived sum-rate expressions, a power allocation scheme that can ensure fairness among the users in the system is also considered. Then the impact of the main system parameters on the performance of the considered schemes are examined and investigated. Results provided in this paper show that CI scheme outperforms the ZF schemes for the same system parameters.

Notations: $h, \mathbf{h}$, and $\mathbf{H}$ denote a scalar, a vector and a matrix, respectively. $(\cdot)^{H},(\cdot)^{T}$ and $\operatorname{diag}($.$) denote conjugate$ transposition, transposition and diagonal of a matrix, respectively. $\mathcal{E}[$.$] denotes average operation. [\mathbf{h}]_{k}$ denotes the $k^{t h}$ 
element in $\mathbf{h},|$.$| denotes the absolute, , and \|.\|^{2}$ denotes the second norm. $\mathbb{C}^{K \times N}$ represents an $\mathrm{K} \times \mathrm{N}$ matrix, and $\mathbf{I}$ denotes the identity matrix.

\section{System Model}

Consider a MU-MIMO system, consisting of a base station, BS, equipped with $N$ antennas communicating with $K$ single antenna users, where $N \geq K$, in same timefrequency resource. All the channels in this paper are modeled as independent identically distributed (i.i.d) Rayleigh fading channels. The channel matrix between the BS and the $K$ users is denoted by $\mathbf{H} \in \mathbb{C}^{K \times N}$, which can be represented as $\mathbf{H}=\mathbf{D}^{1 / 2} \mathbf{H}_{1}$ where $\mathbf{H}_{1} \in \mathbb{C}^{K \times N}$ contains i.i.d $\mathcal{C N}(0,1)$ entries which represent small scale fading coefficients and $\mathbf{D} \in \mathbb{C}^{K \times K}$ is a diagonal matrix with $[\mathbf{D}]_{k k}=\varpi_{k}$ which represent the path-loss attenuation $\varpi_{k}=d_{k}^{-m}, d_{k}$ is the distance between the BS and the $k^{t h}$ user and $m$ is the path loss exponent. The channel vector from the BS to a user $k$ is denoted by $\mathbf{h}_{k}$. It is also assumed that the signal is equiprobably drawn from a $M$-PSK constellation. Considering down-link scenario, and the data symbol vector is drawn from a normalized PSK modulation constellation and denoted as $\mathbf{s} \in \mathbb{C}^{K \times 1}$ [22]. The received signal at the $k^{t h}$ user in this system can be written as,

$$
y_{k}=\mathbf{h}_{k} \mathbf{W} \mathbf{s}+n_{k}
$$

where $\mathbf{h}_{k}$ is the channel vector from the BS to user $k, \mathbf{W}$ is the precoding matrix, $n_{k}$ is the additive white Gaussian noise $(\mathrm{AWGN})$ at the $k^{t h}$ user, $n_{k} \sim \mathcal{C N}\left(0, \sigma_{k}^{2}\right)$.

The achievable rate for the $k$-th user in general MU-MIMO systems is given by [23], [24]

$$
\begin{gathered}
R_{k}=\log _{2} M-\log _{2} e \\
-\frac{1}{M^{N}} \sum_{m=1}^{M^{N}} \mathcal{E}_{\mathbf{h}, n_{k}}\left\{\log _{2} \sum_{i=1}^{M^{N}} e^{\frac{-\left\|\mathbf{h}_{k} \mathbf{W} \boldsymbol{s}_{m, i}+n_{k}\right\|^{2}}{\sigma_{k}^{2}}}\right\}
\end{gathered}
$$

where $\mathbf{s}_{m, i}=\mathbf{s}_{m}-\mathbf{s}_{i}, \mathbf{s}_{m}$ and $\mathbf{s}_{i}$ contain symbols taken from the $M$ signal constellation. In the following, the average sum rate is derived for $\mathrm{ZF}$ and $\mathrm{CI}$ precoding schemes as follows.

\section{ZERO FORCING PRECODING}

In this case $\mathrm{ZF}$ precoding technique is implemented at the BS. Therefore, the precoding matrix can be written as [22], [25],

$$
\mathbf{W}^{Z F}=\beta \mathbf{H}^{H}\left(\mathbf{H H}^{H}\right)^{-1},
$$

where $\beta$ is the scaling factor to meet the transmit power constraint. The received signal at the $k^{t h}$ user now can be written as,

$$
\begin{aligned}
y_{k} & =\beta \mathbf{h}_{k} \mathbf{H}^{H}\left(\mathbf{H H}^{H}\right)^{-1} \mathbf{s}+n_{k}, \\
& =\beta[s]_{k}+n_{k} .
\end{aligned}
$$

Consequently, the rate can be written as

$$
\bar{R}_{k}^{Z F}=\log _{2} M-\log _{2} e
$$

$$
-\frac{1}{M^{N}} \sum_{m=1}^{M^{N}} \mathcal{E}_{\mathbf{H}, n_{k}}\left\{\log _{2} \sum_{i=1}^{M^{N}} e^{\frac{-\left|\beta\left[\mathbf{s}_{m, i}\right]_{k}+n_{k}\right|^{2}}{\sigma_{k}^{2}}}\right\}
$$

By taking the term $e^{\frac{-\left|\beta\left[\mathbf{s}_{m, j}\right]_{k}+n_{k}\right|^{2}}{\sigma_{k}^{2}}}$ out, (5) can be expressed as

$$
\begin{aligned}
\bar{R}_{k}{ }^{Z F}= & \log _{2} M-\log _{2} e \\
& -\frac{1}{M^{N}} \sum_{m=1}^{M^{N}} \mathcal{E}_{\mathbf{H}, n_{k}}\left\{\left(\Upsilon+\log _{2}(1+\Xi)\right)\right\},
\end{aligned}
$$

where $j \in\left[1, M^{N}\right], \Upsilon=\frac{-\left|\beta\left[\mathbf{s}_{m, j}\right]_{k}+n_{k}\right|^{2}}{\sigma_{k}^{2}} \log _{2} e$ and $\Xi=\sum_{i=1, i \neq j}^{M^{N}} e^{\frac{-\left|\beta\left[\mathbf{s}_{m, i}\right]_{k}+n_{k}\right|^{2}+\left|\beta\left[\mathbf{s}_{m, j}\right]_{k}+n_{k}\right|^{2}}{\sigma_{k}^{2}}}$

The scaling factor is given by, $\beta=\sqrt{\frac{p}{\mathbf{s}^{H}\left(\mathbf{H H}^{H}\right)^{-1} \mathbf{s}}}$ [22], [25], where $p$ is the power transmission. For fair comparison, in this paper we consider constant power scaling factor. It was shown that, the term, $X=\frac{\mathbf{s}^{H} \Sigma^{-1} \mathbf{s}}{\mathbf{s}^{H}\left(\mathbf{H H}^{H}\right)^{-1} \mathbf{s}}$, follows Gamma distribution [26], where $\Sigma$ is the covariance of the Wishart matrix $\left(\mathbf{H H}^{H}\right)$. Therefore, average scaling factor can be given by $\beta=\frac{\sqrt{\frac{p}{\mathrm{~s}^{H} \Sigma-\mathrm{s}_{\mathrm{s}}}} \Gamma\left(\frac{3}{2}-K+N\right)}{K \sqrt{K}(N-K) !}$.

To derive the average sum rate in this case, firstly we need to derive the average of the $\Upsilon$ term in (6), which can be obtained as

$$
\begin{aligned}
\mathcal{E}_{\mathbf{H}, n_{k}}\left\{\frac{-\left|\beta\left[\mathbf{s}_{m, j}\right]_{k}+n_{k}\right|^{2}}{\sigma_{k}^{2}} \log _{2} e\right\}= \\
\left\{-\left(\left|\beta\left[\mathbf{s}_{m, j}\right]_{k}\right|^{2}+\sigma_{k}^{2}\right) \frac{\log _{2} e}{\sigma_{k}^{2}}\right\} .
\end{aligned}
$$

In order to calculate the average of the last term in (6), using Jensen inequality, the upper bound can be calculated as

$$
\begin{gathered}
\mathcal{E}_{\mathbf{H}, n_{k}}\left\{\log _{2}\left(1+\sum_{i=1, i \neq j}^{M^{N}} e^{\frac{-\left|\beta\left[\mathbf{s}_{m, i}\right]_{k}+n_{k}\right|^{2}+\left|\beta\left[\mathbf{s}_{m, j}\right]_{k}+n_{k}\right|^{2}}{\sigma_{k}^{2}}}\right)\right\} \\
\log _{2}\left(1+\mathcal{E}_{\mathbf{H}, n_{k}}\left\{\sum_{i=1, i \neq j}^{M^{N}} e^{\frac{-\left|\beta\left[\mathbf{s}_{m, i}\right]_{k}+n_{k}\right|^{2}+\left|\beta\left[\mathbf{s}_{m, j}\right]_{k}+n_{k}\right|^{2}}{\sigma_{k}^{2}}}\right\}\right) .
\end{gathered}
$$

Since $n_{k}$ has Gaussian distribution, the average over $n_{k}$ can be derived as

$$
\mathcal{E}_{n_{k}}\left\{e^{\frac{-\left|\beta\left[\mathbf{s}_{m, i}\right]_{k}+n_{k}\right|^{2}+\left|\beta\left[\mathbf{s}_{m, j}\right]_{k}+n_{k}\right|^{2}}{\sigma_{k}^{2}}}\right\}=
$$




$$
\frac{1}{\pi \sigma_{k}^{2}} \int_{n_{k}} e^{-\frac{\left|\beta\left[\mathbf{s}_{m, i}\right]_{k}+n_{k}\right|^{2}-\left|\beta\left[\mathbf{s}_{m, j}\right]_{k}+n_{k}\right|^{2}+\left|n_{k}\right|^{2}}{\sigma_{k}^{2}}} d n_{k} .
$$

Using the integrals of exponential function in [27], we can find

$$
\begin{gathered}
\mathcal{E}_{n_{k}}\left\{e^{\frac{-\left|\beta\left[\mathbf{s}_{m, i}\right]_{k}+n_{k}\right|^{2}+\left|\beta\left[\mathbf{s}_{m, j}\right]_{k}+n_{k}\right|^{2}}{\sigma_{k}^{2}}}\right\}= \\
e^{-\frac{\left|\beta\left[\mathbf{s}_{m, i}\right]_{k}\right|^{2}-\left|\beta\left[\mathbf{s}_{m, j}\right]_{k}\right|^{2}}{2 \sigma^{2}}}
\end{gathered}
$$

Theorem 1. The total sum rate of the ZF transmission scheme in MU-MIMO systems under PSK signaling can be calculated by

$$
R^{Z F}=\sum_{k=1}^{K} \bar{R}_{k}^{Z F}
$$

where $\bar{R}_{k}^{Z F}$ is given by (12) shown at the top of next page.

In case the users' locations are randomly distributed, the average sum-rate with respect to each user location can be calculated easily by averaging the derived sum-rate over all possible user locations.

\section{Constructive Interference Precoding}

The concept of the CI has been widely studied in MUMIMO systems [9], [28], [29]. For more details about the CI, we refer the reader to the aforementioned works in this paper. In this section, we analyze the performance of CI precoding technique, for the first time. The precoding matrix in the CI can be written as [22],

$$
\mathbf{W}^{C I} \boldsymbol{s}=\frac{1}{K} \beta \mathbf{H}^{H}\left(\mathbf{H} \mathbf{H}^{H}\right)^{-1} \operatorname{diag}\left\{\mathbf{V}^{-1} \mathbf{u}\right\} \mathbf{s},
$$

where $\beta$ is the scaling factor to meet the transmit power constraint, which can be expressed as $\beta=\sqrt{\frac{p}{\mathbf{u}^{H} \mathbf{V}^{-1} \mathbf{u}}}$, $\mathbf{1}^{H} \mathbf{u}=\mathbf{1}$ and $\mathbf{V}=\operatorname{diag}\left(\mathbf{s}^{H}\right)\left(\mathbf{H H}^{H}\right)^{-1} \operatorname{diag}(\mathbf{s})$. In this scenario for the sake of comparison, the normalization constant $\beta$ is considered to be constant. Therefore, the normalization constant can bee written as $\beta=\sqrt{\frac{p}{\mathbf{u}^{H} \operatorname{diag}\left(\mathbf{s}^{H}\right)^{-1} N \boldsymbol{\Sigma}(\operatorname{diag}(\mathbf{s}))^{-1} \mathbf{u}}}$. The received signal at the $k^{t h}$ user now can be written as,

$$
\begin{gathered}
y_{k}=\frac{\beta}{K} \mathbf{h}_{k} \mathbf{H}^{H}\left(\mathbf{H} \mathbf{H}^{H}\right)^{-1} \operatorname{diag}\left\{\mathbf{V}^{-1} \mathbf{u}\right\} \mathbf{s}+n_{k}, \\
=\frac{\beta}{K} \mathbf{a}_{k}\left(\operatorname{diag}\left(\mathbf{s}^{H}\right)\right)^{-1} \mathbf{H} \mathbf{H}^{H}(\operatorname{diag}(\mathbf{s}))^{-1} \mathbf{u}[\boldsymbol{s}]_{k}+n_{k}
\end{gathered}
$$

where $\mathbf{a}_{k}$ is a $1 \times K$ vector all the elements of this vector are zeros except the $k^{\text {th }}$ element is one. Following the principles of $\mathrm{CI}$, the rate at the user $k$ can be written as,

$\bar{R}_{k}^{C I}=\log _{2} M-\log _{2} e$

$$
-\frac{1}{M^{N}} \sum_{m=1}^{M^{N}} \mathcal{E}_{\mathbf{H}, n_{k}}\left\{\left(\Upsilon+\log _{2}(1+\Xi)\right)\right\}
$$

where $\Upsilon=\frac{-\left|\mathbf{h}_{k} \mathbf{W}^{C I} \boldsymbol{s}_{m, j}+n_{k}\right|^{2} \log _{2} e}{\sigma_{k}^{2}}$ and

$\Xi=\sum_{i=1, i \neq j}^{M^{N}} e^{\frac{-\left|\mathbf{h}_{k} \mathbf{W}^{C I} \boldsymbol{s}_{m, i}+n_{k}\right|^{2}+\left|\mathbf{h}_{k} \mathbf{W}^{C I} \boldsymbol{s}_{m, j}+n_{k}\right|^{2}}{\sigma_{k}^{2}}}$.

To start with, the average of $\Upsilon$ term can be obtained as

$$
\begin{aligned}
& \mathcal{E}_{\mathbf{H}, n_{k}}\left\{\frac{-\left|\frac{\beta}{K}\left[\operatorname{diag}\left\{\mathbf{V}^{-1} \mathbf{u}\right\} \boldsymbol{s}_{m, j}\right]_{k}+n_{k}\right|^{2}}{\sigma_{k}^{2}} \log _{2} e\right\}= \\
& \mathcal{E}_{\mathbf{H}}\left\{-\left(\left|\frac{\beta}{K} \mathbf{a}_{k} \operatorname{diag}\left\{\mathbf{V}^{-1} \mathbf{u}\right\}\left[\boldsymbol{s}_{m, j}\right]_{k}\right|^{2}+\sigma_{k}^{2}\right) \frac{\log _{2} e}{\sigma_{k}^{2}}\right\} .
\end{aligned}
$$

In [30] it was shown that, the term $Z=$ $\frac{\mathbf{a}_{k}\left(\operatorname{diag}\left(\mathbf{s}^{H}\right)\right)^{-1}\left(\mathbf{H H}^{H}\right)(\operatorname{diag}(\mathbf{s}))^{-1} \mathbf{u}\left[\boldsymbol{s}_{m, j}\right]_{k}}{\mathbf{a}_{k}\left(\operatorname{diag}\left(\mathbf{s}^{H}\right)\right)^{-1}(\mathbf{\Sigma})(\operatorname{diag}(\mathbf{s}))^{-1} \mathbf{u}\left[\boldsymbol{s}_{m, j}\right]_{k}}$ has Gamma distribution with $N$ degrees of freedom. Consequently the average of (17) can be found as in (18), shown at the top of next page.

In order to calculate the average of the last term in (16), using Jensen inequality, the upper bound can be calculated as follows. Since $n_{k}$ has Gaussian distribution, using similar steps as in the previous section, the average over $n$ can be obtained as

$$
\begin{aligned}
& \mathcal{E}_{n_{k}}\left\{e^{\frac{-\left|\mathbf{h}_{k} \mathbf{w}^{C I} \boldsymbol{s}_{m, i}+n_{k}\right|^{2}+\left|\mathbf{h}_{k} \mathbf{w}^{C I} \boldsymbol{s}_{m, j}+n_{k}\right|^{2}}{\sigma_{k}^{2}}}\right\} \\
= & e^{-\frac{\left|\frac{\beta}{K} \mathbf{a}_{k} \operatorname{diag}\left\{\mathbf{v}^{-1} \mathbf{u}\right\}\left[\boldsymbol{s}_{m, i}\right]_{k}\right|^{2}-\left|\frac{\beta}{K} \mathbf{a}_{k} \operatorname{diag}\left\{\mathbf{v}^{-1} \mathbf{u}\right\}\left[s_{m, j}\right]_{k}\right|^{2}}{2 \sigma_{k}^{2}}} .
\end{aligned}
$$

Now, to obtain the average over $\mathbf{H}$, (19) can be expressed as,

$$
\begin{gathered}
\mathcal{E}_{\mathbf{H}}\left\{e^{-\frac{\left|\frac{\beta}{K} \mathbf{a}_{k} \operatorname{diag}\left\{\mathbf{v}^{-1} \mathbf{u}\right\}\left[s_{m, i}\right]_{k}\right|^{2}-\left|\frac{\beta}{K} \mathbf{a}_{k} \operatorname{diag}\left\{\mathbf{v}^{-1} \mathbf{u}\right\}\left[s_{m, j}\right]_{k}\right|^{2}}{2 \sigma_{k}^{2}}}\right\} \\
=\mathcal{E}_{\mathbf{H}}\left\{e^{-\frac{\chi_{m, i}}{2 \sigma_{k}^{2}}}\right\} .
\end{gathered}
$$

where $\chi_{m, i}=\left|\frac{\beta\left(\mathbf{a}_{k}\left(\operatorname{diag}\left(\mathbf{s}^{H}\right)\right)^{-1} \Sigma(\operatorname{diag}(\mathbf{s}))^{-1} \mathbf{u}\right)}{K} X\left[\boldsymbol{s}_{m, i}\right]_{k}\right|^{2}-$ $\left|\frac{\beta\left(\mathbf{a}_{k}\left(\operatorname{diag}\left(\mathbf{s}^{H}\right)\right)^{-1} \Sigma(\operatorname{diag}(\mathbf{s}))^{-1} \mathbf{u}\right)}{K} X\left[\boldsymbol{s}_{m, j}\right]_{k}\right|^{2}, \quad X \quad=$ $\frac{\mathbf{a}_{k}\left(\operatorname{diag}\left(\mathbf{s}^{H}\right)\right)^{-1}\left(\mathbf{H H}^{H}\right)(\operatorname{diag}(\mathbf{s}))^{-1} \mathbf{u}}{\mathbf{a}_{k}\left(\operatorname{diag}\left(\mathbf{s}^{H}\right)\right)^{-1} \Sigma(\operatorname{diag}(\mathbf{s}))^{-1} \mathbf{u}}$.

The distribution of the $\chi_{m, i}$ is approximated to Gamma distribution [9], [26]. Therefore the average in (20) can be found as in (21), shown at the top of next page, where $\xi=\left(\left|\left[\boldsymbol{s}_{m, i}\right]_{k}\right|^{2}-\left|\left[\boldsymbol{s}_{m, j}\right]_{k}\right|^{2}\right), \quad c_{k}=$ $\frac{\beta\left(\mathbf{a}_{k}\left(\operatorname{diag}\left(\mathbf{s}^{H}\right)\right)^{-1} \Sigma(\operatorname{diag}(\mathbf{s}))^{-1} \mathbf{u}\right)}{K}$ and ${ }_{1} \mathrm{~F}_{1}$ is the Hypergeometric function. 


$$
\bar{R}_{k}^{Z F}=\log _{2} M-\log _{2} e-\frac{1}{M^{N}} \sum_{m=1}^{M^{N}}\left\{\left(-\left(\left|\beta\left[\mathbf{s}_{m, j}\right]_{k}\right|^{2}+\sigma_{k}^{2}\right) \frac{\log _{2} e}{\sigma_{k}^{2}}\right)+\log _{2}\left(1+\sum_{i=1, i \neq j}^{M^{N}} e^{-\frac{\left|\beta\left[\mathbf{s}_{m, i}\right]_{k}\right|^{2}-\left|\beta\left[\mathbf{s}_{m, j}\right]_{k}\right|^{2}}{2 \sigma_{k}^{2}}}\right)\right\}
$$

$$
\begin{aligned}
\varrho_{m, i}=-\left(\mathcal{E}_{\mathbf{H}}\right. & \left.\left\{\left|\frac{\beta\left(\mathbf{a}_{k}\left(\operatorname{diag}\left(\mathbf{s}^{H}\right)\right)^{-1}(\boldsymbol{\Sigma})(\operatorname{diag}(\mathbf{s}))^{-1} \mathbf{u}\left[\boldsymbol{s}_{m, j}\right]_{k}\right)}{K} Z\right|^{2}\right\}+\sigma_{k}^{2}\right) \frac{\log _{2} e}{\sigma_{k}^{2}} \\
& =\left(\frac{-\Gamma(2+N)}{\sigma_{k}^{2} \Gamma(N)}\left|\frac{\beta\left(\mathbf{a}_{k}\left(\operatorname{diag}\left(\mathbf{s}^{H}\right)\right)^{-1}(\boldsymbol{\Sigma})(\operatorname{diag}(\mathbf{s}))^{-1} \mathbf{u}\left[\boldsymbol{s}_{m, j}\right]_{k}\right)}{K}\right|^{2}-1\right) \log _{2} e .
\end{aligned}
$$

$$
\begin{aligned}
& \mathcal{E}_{\mathbf{H}}\left\{e^{-\frac{\chi_{m, i}}{2 \sigma^{2}}}\right\}=\Lambda_{m, i}=\left(\frac{2^{\left(\frac{1}{2}(N-K-1)\right)} K^{(N-K+1)}}{(N-K) !}\right)\left(\left(\frac{c_{k}^{2} \xi}{\sigma_{k}^{2}}\right)^{\frac{1}{2}(K-N-1)}\right) \\
& \times\left(\left(c_{k}^{2}\left(\left|\left[\boldsymbol{s}_{m, i}\right]_{k}\right|^{2}\right)\right) \Gamma\left(\frac{1}{2}(N-K+1)\right){ }_{1} \mathrm{~F}_{1}\left(\frac{1}{2}(N-K+1), \frac{1}{2}, \frac{K^{2} \sigma_{k}^{2}}{2 c_{k}^{2} \xi}\right)\right. \\
& -\left(c_{k}^{2}\left(\left|\left[\boldsymbol{s}_{m, j}\right]_{k}\right|^{2}\right)\right) \Gamma\left(\frac{1}{2}(N-K+1)\right){ }_{1} \mathrm{~F}_{1}\left(\frac{1}{2}(N-K+1), \frac{1}{2}, \frac{K^{2} \sigma_{k}^{2}}{2 c_{k}^{2} \xi}\right) \\
& \left.-\sqrt{2} K \sigma_{k}^{2} \sqrt{\frac{\xi}{\sigma_{k}^{2}}} \Gamma\left(\frac{1}{2}(N-K+2)\right){ }_{1} \mathrm{~F}_{1}\left(\frac{1}{2}(N-K+2), \frac{3}{2}, \frac{K^{2} \sigma_{k}^{2}}{2 c_{k}^{2} \xi}\right)\right) .
\end{aligned}
$$

Theorem 2. The total sum rate of the CI transmission scheme in MU-MIMO systems under PSK signaling can be calculated by

$$
R^{C I}=\sum_{k=1}^{K} \bar{R}_{k}^{C I}
$$

where

$$
\begin{aligned}
\bar{R}_{k}^{C I}= & \log _{2} M-\log _{2} e-\frac{1}{M^{N}} \sum_{m=1}^{M^{N}}\left(\varrho_{m, i}\right. \\
& \left.+\left\{\log _{2}\left(1+\sum_{i=1, i \neq j}^{M^{N}} \Lambda_{m, i}\right)\right\}\right) .
\end{aligned}
$$

\section{USERS' FAIRnESS Algorithm}

In this section, based on the derived expressions of the achievable data rate, the fairness problem is formulated. In specific, we propose a power allocation scheme which maximizes the minimum user rate, $R_{k}$, whilst satisfying the total power constraint as in the following expression,

$$
\begin{aligned}
& \max _{p_{k}} \min _{k=1, \ldots K} R_{k} \\
& \text { s.t. } \sum_{k=1}^{K} p_{k} \leq P_{t}
\end{aligned}
$$

where $p_{k}$ is the power allocated for user $k$, and $P_{t}$ is the total power. However, the expression of the data rate in the systems with non-Gaussian signaling is complicated, and this makes the optimization problem in (24) hard to solve using standard optimization solvers. On the other hand, some iterative algorithms can be used to solve the power allocation problem. Therefore, for a given target rate $R_{T}$, we can consider the following problem,

$$
\begin{gathered}
\text { Find } p_{1}, \ldots, p_{K} \\
\text { s.t. } \sum_{k=1}^{K} p_{k} \leq P_{t}, p_{k} \neq 0 \\
R_{k}=R_{T} \quad k=1, . ., K
\end{gathered}
$$

According to the last formula in (25), the optimal objective function value of (24) $\left(R^{*}\right)$ is larger than or equal to $R_{T}$. In addition, it is known that, in communication systems with finite alphabet signaling the rate increases with the power, and there is minimum power value, $p_{m}$, in which the rate reaches its maximum value, and if the power increases beyond this amount, the rate will be constant. Consequently, based on this fact, the target rate $R_{T}$ for the proposed system can be obtained using Bisection-method as explained in Algorithm 1, shown at the top of next page. 

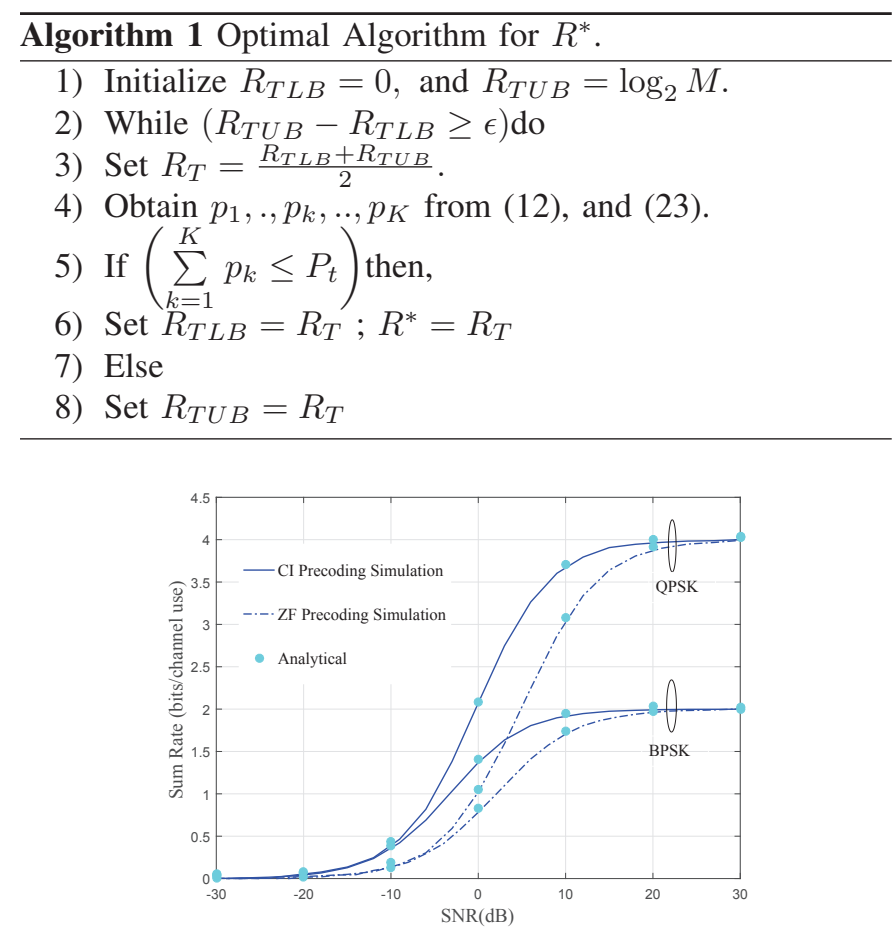

Figure 1: Rate versus SNR with different types of input, when $N=$ $2, K=2$.

\section{Numerical Results}

In this section we present some numerical results of the derived expressions in the previous sections. Monte-Carlo simulations are conducted, in which channel coefficients are randomly generated in each simulation run. Assuming the BS transmission power is $p$, and the users have same noise power $\sigma^{2}$, the SNR can be defined as SNR $=\frac{p}{\sigma^{2}}$. Unless otherwise stated, for sake of comparison, the path loss exponent is chosen to be $m=2.7$, and the distances between the BS and the users are normalized to unit value.

Fig. 1 illustrates sum-rate for the considered transmission schemes, subject to different types of input, BPSK, and QPSK, when $N=2$, and $K=2$. The good agreement between the analytical and simulated results confirms the validity of the analysis in this paper. From this figure, it is evident that the sum rate saturates past a certain SNR, owing to the finite constellation. The sum rate saturates at $2 \mathrm{bits} / \mathrm{s} / \mathrm{Hz}$ in BPSK and at $4 \mathrm{bits} / \mathrm{s} / \mathrm{Hz}$ in QPSK, which can be obtained by the constellation size $M$, number of transmit antennas $N$ and number of users $K$. In addition, the CI technique always outperforms the ZF for a wide range of the SNR. The gap performance between the considered schemes becomes wider in QPSK than that in BPSK, for instance the gain attained by using CI over ZF at $\mathrm{SNR}=10 \mathrm{~dB}$ is approximately $0.2 \mathrm{bits} / \mathrm{s} / \mathrm{Hz}$ in BPSK and around $0.7 \mathrm{bits} / \mathrm{s} / \mathrm{Hz}$ in QPSK.

To capture the impact of number of BS antennas and number of users on the system performance, in Fig. 2 we present the sum-rate for the considered transmission schemes versus SNR, when $N=3$, and $K=3$. Comparing the results in this figure with the results in Fig. 1, it is clear that increasing $N$ and $K$ lead to enhance the system performance. Furthermore, comparing the achievable rates for the BPSK and QPSK in the

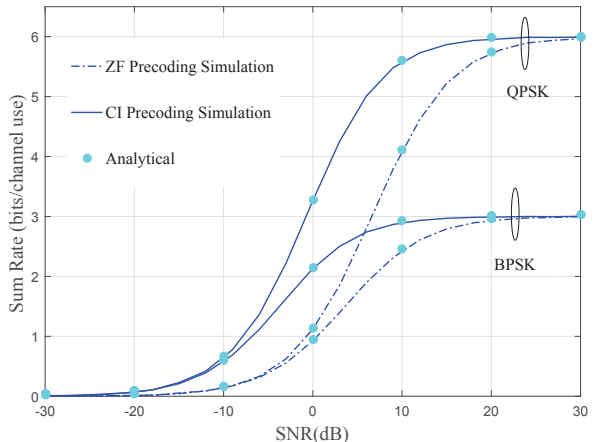

Figure 2: Sum rate versus SNR with different types of input, when $N=$ $3, K=3$.

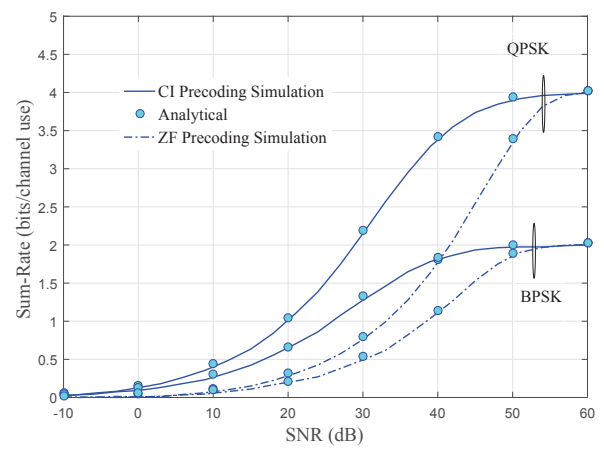

Figure 3: Sum rate versus SNR with BPSK input, when $N=3, K=2$ and the users are randomly distributed.

two scenarios we can notice that, the gap performance between the $\mathrm{CI}$ and $\mathrm{ZF}$ in case $N=K=3$ is wider than that in case $N=K=2$, for instance in Fig. 2 the gain attained by using $\mathrm{CI}$ over $\mathrm{ZF}$ at $\mathrm{SNR}=10 \mathrm{~dB}$ is approximately $0.4 \mathrm{bits} / \mathrm{s} / \mathrm{Hz}$ in BPSK and around $1.7 \mathrm{bits} / \mathrm{s} / \mathrm{Hz}$ in QPSK.

Moreover, in order to investigate the effect of path-loss on the performance of the considering schemes, we plot in Fig. 3 the sum-rate when the users are uniformly distributed inside a circle area with a radius of $60 \mathrm{~m}$, and no user is closer to the BS than $1 \mathrm{~m}$ where the BS is located at the center of this area. It can be seen from these results that, in general, increasing the distance always degrades the system performance, and the sum rate reaches its saturation value at high SNR values, due to larger path-loss.

Finally, Fig. 4 illustrates Jain's fairness index versus the SNR when $N=2, K=2, d_{1}=10 \mathrm{~m}$ and $d_{2}=80 \mathrm{~m}$ in BPSK scenario. The fairness index is defined as [31] $\frac{\left(\sum_{k=1}^{K} R_{k}\right)^{2}}{K \sum_{k=1}^{K} R_{k}^{2}}$, the range of Jain's fairness index is between 0 and 1 , where the maximum achieved when users' rates are equal. From Fig. 4, it can be observed that, the fairness index increases as the SNR increases, and the $\mathrm{CI}$ always achieves higher fairness than $\mathrm{ZF}$ technique. In addition and as anticipated, the proposed power allocation algorithm performs higher fairness than equal power allocation transmission scheme. 


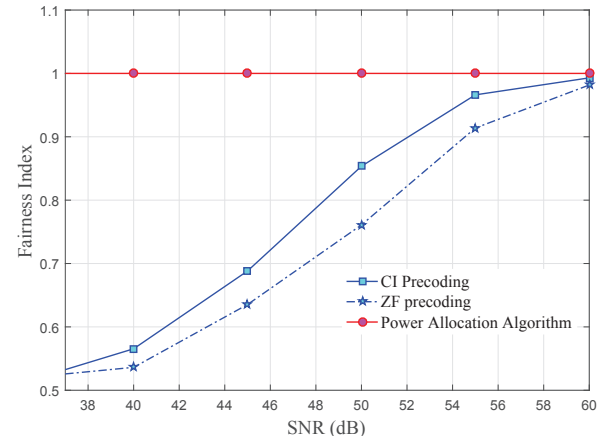

Figure 4: Fairness index versus SNR with BPSK, when $N=2, K=$ $2, d_{1}=10 \mathrm{~m}$ and $d_{2}=80 \mathrm{~m}$

\section{CONCLUSIONS}

In this paper we analyzed the performance of MU-MIMO systems under a PSK input alphabet. New explicit analytical expressions for the average sum rate have been derived for two precoding schemes: 1) ZF precoding technique 2) CI precoding technique. Furthermore, based on the derived expressions a power allocation scheme that can achieve fairness among the users was studied. The results in this work demonstrated that the $\mathrm{CI}$ outperforms the $\mathrm{ZF}$ for same system feature, and the performance gap between the considered schemes depends essentially on the system parameters. In addition, increasing the SNR enhances the sum rate and the fairness among the users, while increasing the distance between the BS and the users degrades the system performance.

\section{REFERENCES}

[1] M. S. John G. Proakis, Digital Communications, Fifth Edition. McGraw-Hill, NY USA, 2008.

[2] C. B. P. Howard Huang and S. Venkatesan, MIMO Communication for cellular Networks. Springer, 2012, 2008.

[3] Y. Wu, C. Xiao, X. Gao, J. D. Matyjas, and Z. Ding, "Linear precoder design for mimo interference channels with finite-alphabet signaling," IEEE Transactions on Communications, vol. 61, no. 9, pp. 3766-3780, September 2013.

[4] W. Wu, K. Wang, W. Zeng, Z. Ding, and C. Xiao, "Cooperative multi-cell mimo downlink precoding with finite-alphabet inputs," IEEE Transactions on Communications, vol. 63, no. 3, pp. 766-779, March 2015.

[5] W. He and C. N. Georghiades, "Computing the capacity of a mimo fading channel under psk signaling," IEEE Transactions on Information Theory, vol. 51, no. 5, pp. 1794-1803, May 2005.

[6] C. Xiao, Y. R. Zheng, and Z. Ding, "Globally optimal linear precoders for finite alphabet signals over complex vector gaussian channels," IEEE Transactions on Signal Processing, vol. 59, no. 7, pp. 3301-3314, July 2011.

[7] W. Zeng, C. Xiao, M. Wang, and J. Lu, "Linear precoding for finitealphabet inputs over mimo fading channels with statistical csi," IEEE Transactions on Signal Processing, vol. 60, no. 6, pp. 3134-3148, June 2012.

[8] M. Wang, W. Zeng, and C. Xiao, "Linear precoding for mimo multiple access channels with finite discrete inputs," IEEE Transactions on Wireless Communications, vol. 10, no. 11, pp. 3934-3942, November 2011.

[9] C. Masouros and E. Alsusa, "Dynamic linear precoding for the exploitation of known interference in mimo broadcast systems," IEEE Transactions on Wireless Communications, vol. 8, no. 3, pp. 1396-1404, March 2009.

[10] C. Masouros and G. Zheng, "Exploiting known interference as green signal power for downlink beamforming optimization," IEEE Transactions on Signal Processing, vol. 63, no. 14, pp. 3628-3640, July 2015.
[11] S. Timotheou, G. Zheng, C. Masouros, and I. Krikidis, "Exploiting constructive interference for simultaneous wireless information and power transfer in multiuser downlink systems," IEEE Journal on Selected Areas in Communications, vol. 34, no. 5, pp. 1772-1784, May 2016.

[12] M. R. A. Khandaker, C. Masouros, and K. K. Wong, "Constructive interference based secure precoding: A new dimension in physical layer security," IEEE Transactions on Information Forensics and Security, vol. 13, no. 9, pp. 2256-2268, Sept 2018.

[13] E. Alsusa and C. Masouros, "Adaptive code allocation for interference management on the downlink of ds-cdma systems," IEEE Transactions on Wireless Communications, vol. 7, no. 7, pp. 2420-2424, July 2008.

[14] K. L. Law, C. Masouros, and M. Pesavento, "Transmit precoding for interference exploitation in the underlay cognitive radio z-channel," IEEE Transactions on Signal Processing, vol. 65, no. 14, pp. 36173631, July 2017.

[15] A. Li and C. Masouros, "Hybrid analog-digital millimeter-wave mumimo transmission with virtual path selection," IEEE Communications Letters, vol. 21, no. 2, pp. 438-441, Feb 2017.

[16] P. V. Amadori and C. Masouros, "Constant envelope precoding by interference exploitation in phase shift keying-modulated multiuser transmission," IEEE Transactions on Wireless Communications, vol. 16, no. 1, pp. 538-550, Jan 2017.

[17] S. Timotheou, G. Zheng, C. Masouros, and I. Krikidis, "Exploiting constructive interference for simultaneous wireless information and power transfer in multiuser downlink systems," IEEE Journal on Selected Areas in Communications, vol. 34, no. 5, pp. 1772-1784, May 2016.

[18] F. A. Khan, C. Masouros, and T. Ratnarajah, "Interference-driven linear precoding in multiuser miso downlink cognitive radio network," IEEE Transactions on Vehicular Technology, vol. 61, no. 6, pp. 2531-2543, July 2012.

[19] P. V. Amadori and C. Masouros, "Large scale antenna selection and precoding for interference exploitation," IEEE Transactions on Соттиnications, vol. 65, no. 10, pp. 4529-4542, Oct 2017.

[20] F. Liu, C. Masouros, P. V. Amadori, and H. Sun, "An efficient manifold algorithm for constructive interference based constant envelope precoding," IEEE Signal Processing Letters, vol. 24, no. 10, pp. 1542-1546, Oct 2017.

[21] C. Masouros, M. Sellathurai, and T. Ratnarajah, "Vector perturbation based on symbol scaling for limited feedback miso downlinks," IEEE Transactions on Signal Processing, vol. 62, no. 3, pp. 562-571, Feb 2014.

[22] A. Li and C. Masouros, "Interference exploitation precoding made practical: Optimal closed-form solutions for psk modulations," IEEE Transactions on Wireless Communications, pp. 1-1, 2018.

[23] Y. Wu, C. Xiao, X. Gao, J. D. Matyjas, and Z. Ding, "Linear precoder design for mimo interference channels with finite-alphabet signaling," IEEE Transactions on Communications, vol. 61, no. 9, pp. 3766-3780, September 2013.

[24] Y. Wu, M. Wang, C. Xiao, Z. Ding, and X. Gao, "Linear precoding for mimo broadcast channels with finite-alphabet constraints," IEEE Transactions on Wireless Communications, vol. 11, no. 8, pp. 29062920, August 2012.

[25] R. Zhang, L. Yang, and L. Hanzo, "Error probability and capacity analysis of generalised pre-coding aided spatial modulation," IEEE Transactions on Wireless Communications, vol. 14, no. 1, pp. 364-375, Jan 2015.

[26] D. Lee, "Performance analysis of zero-forcing-precoded scheduling system with adaptive modulation for multiuser-multiple input multiple output transmission," IET Communications, vol. 9, no. 16, pp. 20072012, 2015.

[27] M. Abramowitz and I. A. Stegun, Handbook of Mathematical Functions With Formulas, Graphs, and Mathematical Tabl, Washington,D.C.: U.S. Dept. Commerce, 1972.

[28] C. Masouros, T. Ratnarajah, M. Sellathurai, C. B. Papadias, and A. K. Shukla, "Known interference in the cellular downlink: a performance limiting factor or a source of green signal power?" IEEE Communications Magazine, vol. 51, no. 10, pp. 162-171, October 2013.

[29] G. Zheng, I. Krikidis, C. Masouros, S. Timotheou, D. A. Toumpakaris, and Z. Ding, "Rethinking the role of interference in wireless networks," IEEE Communications Magazine, vol. 52, no. 11, pp. 152-158, Nov 2014.

[30] R. J. Muirhead, Aspects of Multivariate Statistical Theory, 1982.

[31] H. B. Jung and D. K. Kim, "Power control of femtocells based on maxmin fairness in heterogeneous networks," IEEE Communications Letters, vol. 17, no. 7, pp. 1372-1375, July 2013. 